\title{
EXPONENTIATED PARETO DISTRIBUTION: A BAYES STUDY UTILIZING MCMC TECHNIQUE UNDER UNIFIED HYBRID CENSORING SCHEME
}

\author{
M. G. M. Ghazal ${ }^{1}$ and Q. A. Shihab ${ }^{2}$ \\ [12pt] ${ }^{1,2}$ Mathematics Department, Faculty of Science, Minia University, Minia, Egypt.
}

Received 4/3/2018 Revised 25/3/2018 Accepted 6/7/2018

Abstract: This article aims to study the problem of point and interval estimations of the exponentiated Pareto distribution utilizing unified hybrid censored scheme (HCS). We utilize three methods, including the maximum likelihood, parametric bootstrap and Bayes of estimating the unknown parameters, reliability, hazard rate functions and coefficient of variation. Furthermore, Markov Chain Monte Carlo samples utilizing importance sampling scheme are utilized to generate the Bayes estimates and the credible intervals for unknown quantities. The findings of Bayes method computed using balanced loss function. The suggested methods can be understood by analysing a set of real data.

MSC: $62 \mathrm{~N} 02,62 \mathrm{~F} 15$.

\section{Introduction}

In life-testing and reliability studies, it is prefered to use a censoring scheme that can balance the total time spent on the experiment, the number of units utilized in the experiment and the efficiency of statistical analysis under the findings of the experiment. Type-I (time) and Type-II (failure) censoring schemes are deemed to be two of the most known censoring schemes. In Type-I, the experimental time is fixed and the number of observed failures is random. On the other hand, in Type-II the number of observed failures is fixed and the experimental time is random. More above, the two types do not allow units removal at certain points, other than the expermient terminal point. In order to overcome the challenge of inflexibility in the traditional Type I and Type II censoring schemes, more comprehensive censoring schemes are proposed. Many authors presented several hybrid censoring schemes (HCS), including Type-I HCS (Epstein [1]), Type-II HCS (Childs et al. [2]) and generalized HCS, where it contains generalized Type-I and Type-II HCS (see, Chandrasekar et al. [3]). Generalized HCS have some drawbacks, for instance in generalized Type-I HCS the experiment terminated when the same time or before $T$, so we cannot ensure observing $r$ failures. On the other hand, in the generalized Type-II HCS, we cannot observe any failure at all or observe only a few number of failures until the pre-fixed time $T_{2}$. To overcome the problems of these schemes, Balakrishnan et al. [4] presented a unified HCS which can be spicified as follows:

Fix integers $r, k \in\{1, \ldots, n\}$ where $k<r<n$ and $T_{1}, T_{2} \in(0, \infty)$ where $T_{2}>T_{1}$. When $k^{t h}$ failure occurs before time $T_{1}$, the experiment is terminated at $\min \left\{\max \left\{X_{r: n}, T_{1}\right\}, T_{2}\right\}$. When $k^{\text {th }}$ failure occurs between $T_{1}$ and $T_{2}$, the experiment is terminated at $\min \left\{X_{r: n}, T_{2}\right\}$ and when $k^{t h}$ failure occurs after time $T_{2}$, the experiment is terminated at $X_{k: n}$. Therefore, depending on this censoring scheme, we can ensure that the experiment would be finished at most in time $T_{2}$ with at least $k$ failures and if not, we can ensure exactly $k$ failures. Thence, based on this unified HCS, we get the following six cases:

Case I: $0<x_{k: n}<x_{r: n}<T_{1}<T_{2}$, the experiment terminated when $T_{1}$.

Case II: $0<x_{k: n}<T_{1}<x_{r: n}<T_{2}$, the experiment terminated when $x_{r: n}$.

Case III: $0<x_{k: n}<T_{1}<T_{2}<x_{r: n}$, the experiment terminated when $T_{2}$. 
Case IV: $0<T_{1}<x_{k: n}<x_{r: n}<T_{2}$, the experiment terminated when $x_{r: n}$.

Case V: $0<T_{1}<x_{k: n}<T_{2}<x_{r: n}$, the experiment terminated when $T_{2}$.

Case VI: $0<T_{1}<T_{2}<x_{k: n}<x_{r: n}$, the experiment terminated when $x_{k: n}$.

For more information about generalized HCS and unified HCS see, Epstein [1], Chandrasekar et al. [3], Balakrishnan et al. [4], Balakrishnan and Kundu [5], Panahi and Sayyareh [6], Shafay [7], Mahmoud and Ghazal [8] and Ghazal and Hasaballah [9].

Gupta et al. [10] proposed that the exponentiated Pareto distribution (EPD) can be utilized quite effectively in analyzing many lifetime data. Ali et al. [11,12] studied several exponentiated distributions, including, EPD and discussed their properties. They indicated that the EPD is a good fit to the tail-distribution for NASDAQ data. Shawky and AbuZinadah [13] examined different estimators of the unknown parameters based on EPD. Afify [14] discussed estimation of the EPD under type I and type II censoring schemes. Finally, Mahmoud et al. [15] explored the estimation of parameters for the EPD depending on progressively type-II right censored data. The probability density function (PDF) and the cumulative distribution function (CDF) of the EPD with two shape parameters $\beta$ and $\theta$ are given, respectively, by

$$
f(x ; \beta, \theta)=\beta \theta(1+x)^{-(\beta+1)}\left(1-(1+x)^{-\beta}\right)^{\theta-1}, \quad x>0, \quad \beta, \theta>0,
$$

and

$$
F(x ; \beta, \theta)=\left(1-(1+x)^{-\beta}\right)^{\theta} .
$$

Futher, the reliability $R(t)$ and hazard rate $H(t)$ functions of the $\operatorname{EPD}(\beta, \theta)$ at time $t$ are given, respectively, by

$$
R(t)=1-\left(1-(1+t)^{-\beta}\right)^{\theta}, \quad t>0,
$$

and

$$
H(t)=\frac{\beta \theta(1+t)^{-(\beta+1)}\left(1-(1+t)^{-\beta}\right)^{\theta-1}}{1-\left(1-(1+t)^{-\beta}\right)^{\theta}}, \quad t>0 .
$$

Many scientific areas such as engineering, economics, biology, and psychology have utilized the coefficient of variation $(C V(X))$ in queueing and reliability theory (see, Sharma and Krishna [16]). $C V(X)$ of the $\operatorname{EPD}(\beta, \theta)$ is

$$
C V(X)=\frac{\sqrt{\operatorname{Var}(X)}}{E(X)}, \quad E(X)>0
$$

where $E(X)$ and $\operatorname{Var}(X)$ are given, respectively, by

$$
\begin{gathered}
E(X)=\theta B\left(1-\frac{1}{\beta}, \theta\right)-1, \quad \beta>1, \\
\operatorname{Var}(X)=\theta B\left(1-\frac{2}{\beta}, \theta\right)-\theta^{2}\left(B\left(1-\frac{1}{\beta}, \theta\right)\right)^{2}, \quad \beta>2,
\end{gathered}
$$

where $B(a, b)=\frac{\Gamma(a) \Gamma(b)}{\Gamma(a+b)}$ is Beta function. According to Equation (1.5), the $C V(X)$ for the $\operatorname{EPD}(\beta, \theta)$ is given by

$$
C V(X)=\frac{\sqrt{\theta B\left(1-\frac{2}{\beta}, \theta\right)-\theta^{2}\left(B\left(1-\frac{1}{\beta}, \theta\right)\right)^{2}}}{\theta B\left(1-\frac{1}{\beta}, \theta\right)-1}, \quad \beta>2 .
$$

\section{Estimation of the Parameters}

In the present section, we estimate $\beta$ and $\theta$ through considering the maximum likelihood estimators (MLEs) as well as $R(t), H(t)$ and $C V(X)$. We calculate the observed Fisher information under the likelihood equations $I_{i j}=\frac{\partial^{2} \ell(\underline{x} ; \beta, \theta)}{\partial \phi_{i} \partial \phi_{j}}$ where $\phi_{1}=\beta, \phi_{2}=\theta$ for $i, j=1,2$. Also, we compute asymptotic confidence intervals (CIs) for $\beta, \theta, R(t), H(t)$ and $C V(X)$ utilizing the normality property of corresponding MLEs. 


\subsection{Maximum likelihood estimation}

Assumeing that $X_{1: n}<X_{2: n}<\ldots<X_{n: n}$ be a random sample of size $n$ from $\operatorname{EPD}(\beta, \theta)$, according to the unified HCS described in Section 1, the likelihood function takes the form

$$
L(\underline{x} ; \beta, \theta)=\frac{n !}{(n-D) !} \prod_{i=1}^{D} f\left(x_{i}\right)\left(1-F\left(T^{*}\right)\right)^{n-D},
$$

where

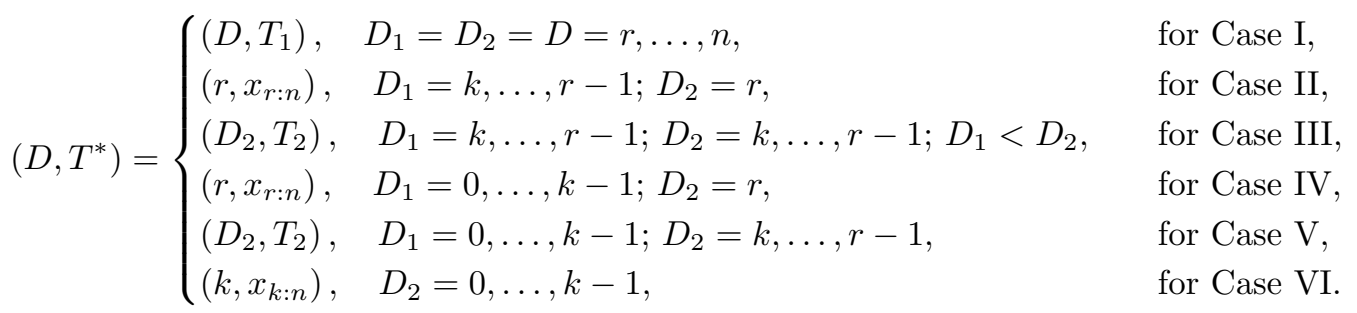

Where, $D_{m}$ indicates the number of failures till time $T_{m}, m=1,2$. Utilizing Equations (1.2) and (1.1) in Equation (2.1) and ignoring the normalized constant, the likelihood function of $\beta$ and $\theta$ is obtained as

$$
\left.L(\underline{x} ; \beta, \theta) \propto \beta^{D} \theta^{D} e^{-(\beta+1) \sum_{i=1}^{D} \ln \left(1+x_{i}\right)} e^{(\theta-1) \sum_{i=1}^{D} \ln \left(1-\left(1+x_{i}\right)^{-\beta}\right.}\right)\left(1-\left(1-\left(1+T^{*}\right)^{-\beta}\right)^{\theta}\right)^{n-D}
$$

The $\log$-likelihood function $\ell(\underline{x} ; \beta, \theta)$ of $\beta$ and $\theta$ is obtained from Equation (2.3) as

$$
\begin{aligned}
\ell(\underline{x} ; \beta, \theta)= & D \ln \beta+D \ln \theta-(\beta+1) \sum_{i=1}^{D} \ln \left(1+x_{i}\right)+(\theta-1) \sum_{i=1}^{D} \ln \left(1-\left(1+x_{i}\right)^{-\beta}\right) \\
& +(n-D) \ln \left(1-\left(1-\left(1+T^{*}\right)^{-\beta}\right)^{\theta}\right) .
\end{aligned}
$$

Differentiating Equation (2.4) with respect to $\beta$ and $\theta$ and equating each result to zero, we obtaine likelihood equations as

$$
\begin{gathered}
\frac{\partial \ell(\underline{x} ; \beta, \theta)}{\partial \beta}=\frac{D}{\beta}-\sum_{i=1}^{D} \ln \left(1+x_{i}\right)+(\theta-1) \sum_{i=1}^{D} \frac{\ln \left(1+x_{i}\right)}{\left(1+x_{i}\right)^{\beta}-1} \\
-\frac{\theta(n-D)\left(1-\left(1+T^{*}\right)^{-\beta}\right)^{\theta-1}\left(1+T^{*}\right)^{-\beta} \ln \left(1+T^{*}\right)}{1-\left(1-\left(1+T^{*}\right)^{-\beta}\right)^{\theta}}=0,
\end{gathered}
$$

and

$$
\frac{\partial \ell(\underline{x} ; \beta, \theta)}{\partial \theta}=\frac{D}{\theta}+\sum_{i=1}^{D} \ln \left(1-\left(1+x_{i}\right)^{-\beta}\right)-\frac{(n-D) \ln \left(1-\left(1+T^{*}\right)^{-\beta}\right)}{\left(1-\left(1+T^{*}\right)^{-\beta}\right)^{-\theta}-1}=0 .
$$

Equations (2.5) and (2.6) cannot be solved analytically for $\beta$ and $\theta$, hence, we utilize numerical methods such as Newton's Raphson method to compute maximum likelihood estimates for $\beta$ and $\theta$, say $\hat{\beta}_{\mathrm{ML}}$ and $\hat{\theta}_{\mathrm{ML}}$, respectively. Consequently, utilizing the invariance property, the MLEs of $R(t), H(t)$ and $C V(X)$ can be computed after substituting $\beta$ and $\theta$ by $\hat{\beta}_{\mathrm{ML}}$ 
and $\hat{\theta}_{\mathrm{ML}}$, as

$$
\left\{\begin{array}{l}
\hat{R}_{\mathrm{ML}}(t)=1-\left(1-(1+t)^{-\hat{\beta}_{\mathrm{ML}}}\right)^{\hat{\theta}_{\mathrm{ML}}}, \\
\hat{H}_{\mathrm{ML}}(t)=\frac{\hat{\beta}_{\mathrm{ML}} \hat{\theta}_{\mathrm{ML}}(1+t)^{-\left(\hat{\beta}_{\mathrm{ML}}+1\right)}\left(1-(1+t)^{-\hat{\beta}_{\mathrm{ML}}}\right)^{\hat{\theta}_{\mathrm{ML}}-1}}{1-\left(1-(1+t)^{-\hat{\beta}_{\mathrm{ML}}}\right)^{\hat{\theta}_{\mathrm{ML}}}}, \\
\widehat{C V}_{\mathrm{ML}}(X)=\frac{\sqrt{\hat{\theta}_{\mathrm{ML}} B\left(1-\frac{2}{\hat{\beta}_{\mathrm{ML}}}, \hat{\theta}_{\mathrm{ML}}\right)-\hat{\theta}_{\mathrm{ML}}^{2}\left(B\left(1-\frac{1}{\hat{\beta}_{\mathrm{ML}}}, \hat{\theta}_{\mathrm{ML}}\right)\right)^{2}}}{\hat{\theta}_{\mathrm{ML}} B\left(1-\frac{1}{\hat{\beta}_{\mathrm{ML}}}, \hat{\theta}_{\mathrm{ML}}\right)-1} .
\end{array}\right.
$$

\subsection{Approximate Interval Estimation}

The Fisher information matrix $I(\beta, \theta)$ is then obtained by expecting $I_{i j}=-\frac{\partial^{2} \ell(\underline{x} ; \beta, \theta)}{\partial \phi_{i} \partial \phi_{j}}$ where $\phi_{1}=\beta, \phi_{2}=\theta$ for $i, j=1,2$. Evidently, obtaining the exact expressions of the above expectation represents a challenge. Accordingly, we extract the approximate asymptotic variance-covariance matrix of MLEs given as

$$
I_{0}(\hat{\beta}, \hat{\theta})=[\hat{V}]=\left(\begin{array}{ll}
-\frac{\partial^{2} \ell(\underline{x} ; \beta, \theta)}{\partial \beta^{2}} & -\frac{\partial^{2} \ell(\underline{x} ; \beta, \theta)}{\partial \beta \partial \theta} \\
-\frac{\partial^{2} \ell(\underline{x} ; \beta, \theta)}{\partial \theta \partial \beta} & -\frac{\partial^{\ell} \ell(\underline{x} ; \beta, \theta)}{\partial \theta^{2}}
\end{array}\right)_{(\hat{\beta}, \hat{\theta})}^{-1}=\left(\begin{array}{cc}
\widehat{\operatorname{Var}}(\hat{\beta}) & \operatorname{Cov}(\hat{\beta}, \hat{\theta}) \\
\operatorname{Cov}(\hat{\beta}, \hat{\theta}) & \widehat{\operatorname{Var}(\hat{\theta})}
\end{array}\right) .
$$

Thus, it can be utilized to calculate the $100(1-\tau) \%$ approximate CIs for the parameters $\beta$ and $\theta$ which are given, respectively, by

$$
\left(\hat{\beta}_{\mathrm{ML}} \mp Z_{\frac{\tau}{2}} \sqrt{\widehat{\operatorname{Var}}\left(\hat{\beta}_{\mathrm{ML}}\right)}\right) \quad, \quad\left(\hat{\theta}_{\mathrm{ML}} \mp Z_{\frac{\tau}{2}} \sqrt{\widehat{\operatorname{Var}}\left(\hat{\theta}_{\mathrm{ML}}\right)}\right),
$$

where $Z_{\frac{\tau}{2}}$ is the percentile of $N(0,1)$ with right-tail probability $\frac{\tau}{2}$.

Moreover, to construct the asymptotic CIs of the reliability, hazard functions and coefficient of variation, we need to compute the variances of them by utilizing the delta method, see Greene [17]. Assume that $A_{1}=\left(\frac{\partial R(t)}{\partial \beta} \frac{\partial R(t)}{\partial \theta}\right)$, $A_{2}=\left(\begin{array}{cc}\frac{\partial H(t)}{\partial \beta} & \frac{\partial H(t)}{\partial \theta}\end{array}\right)$ and $A_{3}=\left(\begin{array}{cc}\frac{\partial C V(X)}{\partial \beta} & \frac{\partial C V(X)}{\partial \theta}\end{array}\right)$, where $\frac{\partial R(t)}{\partial \beta}, \frac{\partial R(t)}{\partial \theta}, \frac{\partial H(t)}{\partial \beta}, \frac{\partial H(t)}{\partial \theta}, \frac{\partial C V(X)}{\partial \beta}$ and $\frac{\partial C V(X)}{\partial \theta}$ are the first derivatives of the $R(t), H(t)$ and $C V(X)$ with respect to the parameters $\beta$ and $\theta$, respectively. The approximate asymptotic variances of $\hat{R}_{\mathrm{ML}}(t), \hat{H}_{\mathrm{ML}}(t)$ and $\widehat{C V}_{\mathrm{ML}}(X)$ can be computed, respectively, by

$$
\widehat{\operatorname{Var}}\left(\hat{R}_{\mathrm{ML}}(t)\right)=\left(A_{1} \hat{V} A_{1}^{T}\right)_{(\hat{\beta}, \hat{\theta})}, \widehat{\operatorname{Var}}\left(\hat{H}_{\mathrm{ML}}(t)\right)=\left(A_{2} \hat{V} A_{2}^{T}\right)_{(\hat{\beta}, \hat{\theta})}, \widehat{\operatorname{Var}}\left(\widehat{C V}{ }_{\mathrm{ML}}(X)\right)=\left(A_{3} \hat{V} A_{3}^{T}\right)_{(\hat{\beta}, \hat{\theta})},
$$

where, $A_{k}^{T}$ is the transpose of $A_{k}, k=1,2,3$. These findings yield the approximate CIs for $R(t), H(t)$ and $C V(X)$ as follows:

$$
\left(\hat{R}_{\mathrm{ML}}(t) \mp Z_{\frac{\tau}{2}} \sqrt{\widehat{\operatorname{Var}}\left(\hat{R}_{\mathrm{ML}}(t)\right)}\right),\left(\hat{H}_{\mathrm{ML}}(t) \mp Z_{\frac{\tau}{2}} \sqrt{\widehat{\operatorname{Var}}\left(\hat{H}_{\mathrm{ML}}(t)\right)}\right),\left(\widehat{C V}_{\mathrm{ML}}(X) \mp Z_{\frac{\tau}{2}} \sqrt{\widehat{\operatorname{Var}}\left(\widehat{C V}_{\mathrm{ML}}(X)\right)}\right) .
$$

\section{Bootstrap confidence intervals}

In the present section, two parametric bootstrap procedures introduced to construct the bootstrap CIs of $\beta, \theta, R(t), H(t)$ and $C V(X)$ : The percentile bootstrap (Boot-p) confidence interval by Efron [18] and the bootstrap-t (Boot-t) confidence interval proposed by Hall [19]. The following algorithms show the estimation of CIs utilizing both methods.

\subsection{Parametric Boot-p}

1. Utilize the original data $\underline{x}=x_{1: n}, \ldots, x_{n: n}$ compute $\hat{\beta}$ and $\hat{\theta}$ via maximizing Equations (2.5) and (2.6).

2. Obtain a bootstrap sample $\underline{x}^{*}=x_{1: n}^{*}, \ldots, x_{n: n}^{*}$ through resampling with replacement.

3. Compute the MLEs depending on the bootstrap sample and indicate this bootstrap estimate by $\hat{\varphi}^{*}(\varphi=\beta, \theta, R(t), H(t)$ or $C V(X))$. 
4. Duplicate 2 and 3 NBoot times, then compute $\hat{\varphi}_{1}^{*}, \hat{\varphi}_{2}^{*}, \ldots, \hat{\varphi}_{\mathrm{NBoot}}^{*}, \hat{\varphi}_{l}^{*}=\left(\hat{\beta}_{l}^{*}, \hat{\theta}_{l}^{*}, \hat{R}_{l}^{*}(t), \hat{H}_{l}^{*}(t)\right.$, $\left.\widehat{C V}_{l}^{*}(X)\right), l=1,2, \ldots$, NBoot.

5. Arrange $\hat{\varphi}_{l}^{*}, l=1,2, \ldots$, NBoot in ascending orders, thence compute $\hat{\varphi}_{(1)}^{*}, \hat{\varphi}_{(2)}^{*}, \ldots, \hat{\varphi}_{(\mathrm{NBoot})}^{*}$.

6. Suppose that $\widehat{G}_{1}(u)=P\left(\hat{\varphi}^{*} \leq u\right)$ be the $\operatorname{CDF}$ of $\hat{\varphi}^{*}$. Idintify $\hat{\varphi}_{\mathrm{NBootP}}=\widehat{G}_{1}^{-1}(u)$ for given $u$. The approximate

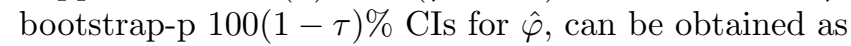

$$
\left(\hat{\varphi}_{\mathrm{NBootP}}\left(\frac{\tau}{2}\right), \hat{\varphi}_{\mathrm{NBootP}}\left(1-\frac{\tau}{2}\right)\right)
$$

\subsection{Parametric Boot-t}

1-3. The same as above-mentioned steps.

4. Based on Equation (2.8) calculate $I^{-1 *}\left(\hat{\beta}^{*}, \hat{\theta}^{*}\right)$.

5. Calculate $T^{* \varphi}$ statistic from $T^{* \varphi}=\frac{\left(\hat{\varphi}^{*}-\hat{\varphi}\right)}{\sqrt{\widehat{\operatorname{Var}}\left(\hat{\varphi}^{*}\right)}}$.

6. Duplicate the procedues 2-5, NBoot times, then calculate $T_{1}^{* \varphi}, T_{2}^{* \varphi}, \ldots, T_{\mathrm{NBoot}}^{* \varphi}$.

7. Sort $T_{1}^{* \varphi}, T_{2}^{* \varphi}, \ldots, T_{\text {NBoot }}^{* \varphi}$ in ascending orders, then calculate the following ordered sequences

$$
T_{(1)}^{* \varphi}, T_{(2)}^{* \varphi}, \ldots, T_{(\mathrm{NBoot})}^{* \varphi} .
$$

8. Suppose that $\widehat{G}_{2}(u)=P\left(T^{*} \leq u\right)$ be the CDF of $T^{*}$. For a given $u$, define

$$
\hat{\varphi}_{\mathrm{NBootT}}=\hat{\varphi}+\widehat{G}_{2}^{-1}(u) \sqrt{\widehat{\operatorname{Var}}\left(\hat{\varphi}^{*}\right)} .
$$

Subsequently, the approximate bootstrap-t $100(1-\tau) \%$ CIs of $\hat{\varphi}$ is given by

$$
\left(\hat{\varphi}_{\mathrm{NBootT}}\left(\frac{\tau}{2}\right), \hat{\varphi}_{\mathrm{NBootT}}\left(1-\frac{\tau}{2}\right)\right) \text {. }
$$

\section{Bayes estimation utilizing MCMC technique}

In the present section, we compute Bayesian estimates and the corresponding credible intervals of $\beta$ and $\theta$ in addition to some lifetime parameters, including $R(t), H(t)$ and $C V(X)$. We suppose that the parameters $\beta$ and $\theta$ are independent and follow the gamma prior distributions

$$
\begin{cases}\pi_{1}(\beta) \propto \beta^{a_{1}-1} e^{-b_{1} \beta}, & \beta>0, \\ \pi_{2}(\theta) \propto \theta^{a_{2}-1} e^{-b_{2} \theta}, & \theta>0 .\end{cases}
$$

Here, all the hyper parameters $a_{1}, a_{2}, b_{1}$ and $b_{2}$ are known and positive. As a consequence, the joint prior distribution $\pi(\beta, \theta)$ for $\beta$ and $\theta$ is

$$
\pi(\beta, \theta) \propto \beta^{a_{1}-1} \theta^{a_{2}-1} e^{-\left(b_{1} \beta+b_{2} \theta\right)} .
$$

The posterior distribution of the parameters $\beta$ and $\theta$ indicated by $\pi^{*}(\beta, \theta \mid \underline{x})$ up to proportionality is determined by combining the likelihood function Equation (2.3) with the prior Equation (4.1) via Bayes' theorem, then $\pi^{*}(\beta, \theta \mid \underline{x})$ is formulated as

$$
\pi^{*}(\beta, \theta \mid \underline{x})=\frac{L(\underline{x} \mid \beta, \theta) \times \pi_{1}(\beta) \times \pi_{2}(\theta)}{\int_{0}^{\infty} \int_{0}^{\infty} L(\underline{x} \mid \beta, \theta) \times \pi_{1}(\beta) \times \pi_{2}(\theta) d \beta d \theta} .
$$

Consequently, we can determine the Bayes estimate of any parameters function, such as $h(\beta, \theta)$, based on squared error loss function, as

$$
E_{\beta, \theta \mid \underline{x}}(h(\beta, \theta \mid \underline{x}))=\frac{\int_{0}^{\infty} \int_{0}^{\infty} h(\beta, \theta \mid \underline{x}) \times L(\underline{x} \mid \beta, \theta) \times \pi_{1}(\beta) \times \pi_{2}(\theta) d \beta d \theta}{\int_{0}^{\infty} \int_{0}^{\infty} L(\underline{x} \mid \beta, \theta) \times \pi_{1}(\beta) \times \pi_{2}(\theta) d \beta d \theta} .
$$


It may be noted that, the computation of (4.4) cannot be solved analytically. It is due to the complicated form of the likelihood function given in Equation (2.3). Consequently, MCMC technique is utilized to generate samples from Equation (4.3). Then, we use samples to calculate the Bayes estimate of $\beta$ and $\theta$ and any function of them such as $R(t), H(t)$ and $C V(X)$ and to construct associated CIs. To apply the MCMC technique, we use the Gibbs within Metropolis sampler which requires the derivation of the complete set of conditional posterior distribution. From $(4.3), \pi^{*}(\beta, \theta \mid \underline{x})$ up to proportionality is formulated as

$$
\begin{aligned}
\pi^{*}(\beta, \theta \mid \underline{x}) \propto & \beta^{R+a_{1}-1} \theta^{R+a_{2}-1} e^{-\beta\left(b_{1}+\sum_{i=1}^{R} \ln \left(1+x_{i}\right)\right)} e^{-b_{2} \theta+(\theta-1) \sum_{i=1}^{R} \ln \left(1-\left(1+x_{i}\right)^{-\beta}\right)} \\
& \times\left(1-\left(1-(1+C)^{-\beta}\right)^{\theta}\right)^{n-R} .
\end{aligned}
$$

From (4.5), the conditional posterior densities of $\beta$ and $\theta$ are given, respectively, by

$$
\begin{gathered}
\pi^{*}(\beta \mid \theta, \underline{x}) \propto \beta^{R+a_{1}-1} e^{-\beta\left(b_{1}+\sum_{i=1}^{R} \ln \left(1+x_{i}\right)\right)}, \\
\left.\pi^{*}(\theta \mid \beta, \underline{x}) \propto \theta^{R+a_{2}-1} e^{-b_{2} \theta+(\theta-1) \sum_{i=1}^{R} \ln \left(1-\left(1+x_{i}\right)^{-\beta}\right.}\right)\left(1-\left(1-(1+C)^{-\beta}\right)^{\theta}\right)^{n-R} .
\end{gathered}
$$

The conditional posterior density of $\beta$ in Equation (4.6) is gamma density with shape parameter $\left(R+a_{1}\right)$ and scale parameter $\left(b_{1}+\sum_{i=1}^{R} \ln \left(1+x_{i}\right)\right)$. Subsequently, samples of $\beta$ can be easily generated utilizing any gamma generating routine. The conditional posterior distribution of $\theta$ in Equation (4.7) can not be present in standard form, but its plot is similar to normal distribution. So, Gibbs sampling is not a straight-forward option. Using Metropolis-Hasting (M-H) sampler is necessary for applying MCMC methodology. The following procedures show the process of the M-H algorithm within Gibbs sampling:

1. For given $t$, start with initial guess $\left(\beta^{0}, \theta^{0}\right)$.

2. Put $j=1$.

3. Generate $\beta^{j}$ from Gamma $\left(R+a_{1},\left(b_{1}+\sum_{i=1}^{R} \ln \left(1+x_{i}\right)\right)\right)$.

4. Use the following M-H algorithm, generate $\theta^{(j)}$ from $\pi_{2}^{*}\left(\theta^{j-1} \mid \beta^{j}, \underline{x}\right)$ with $N\left(\theta^{(j-1)}, \sigma^{2}(\theta)\right)$.

(i) Generate a proposal $\theta^{*}$ from $N\left(\theta^{(j-1)}, \sigma^{2}(\theta)\right)$.

(ii) Compute the acceptance probability

$$
\rho=\min \left[1, \frac{\pi_{2}^{*}\left(\theta^{*} \mid \beta^{j}, \underline{x}\right)}{\pi_{2}^{*}\left(\theta^{j-1} \mid \beta^{j}, \underline{x}\right)}\right] .
$$

(iii) Generate $u$ from a Uniform $(0,1)$ distribution.

(iv) If $u<\rho$. accept the proposal and put $\theta^{(j)}=\theta^{*}$. Otherwise, put $\theta^{(j)}=\theta^{(j-1)}$.

5. Calculate $R(t), H(t)$ and $C V(X)$ as

$$
\left\{\begin{array}{l}
R^{(j)}(t)=1-\left(1-(1+t)^{-\beta^{(j)}}\right)^{\theta^{(j)}} \\
H^{(j)}(t)=\frac{\beta^{(j)} \theta^{(j)}(1+t)^{-\left(\beta^{(j)}+1\right)}\left(1-(1+t)^{-\beta^{(j)}}\right)^{\theta^{(j)}-1}}{1-\left(1-(1+t)^{-\beta^{(j)}}\right)^{\theta^{(j)}}}, \\
C V^{(j)}(X)=\frac{\sqrt{\theta^{(j)} B\left(1-\frac{2}{\beta^{(j)}}, \theta^{(j)}\right)-\left(\theta^{(j)}\right)^{2}\left(B\left(1-\frac{1}{\beta^{(j)}}, \theta^{(j)}\right)\right)^{2}}}{\theta^{(j)} B\left(1-\frac{1}{\beta^{(j)}}, \theta^{(j)}\right)-1} .
\end{array}\right.
$$


6. Put $j=j+1$.

7. Duplicate 3-6, $\mathrm{N}$ times.

For ensuring the convergence and removing the affection of the selection of initial value, we have to discard the first $M$ simulated varieties. Thence the selected samples are $\beta^{(j)}, \theta^{(j)}, R^{(j)}(t), H^{(j)}(t)$ and $C V^{(j)}(X), j=M+1, \ldots, N$, for sufficiently large $N$, compose an approximate posterior sample that is utilized to improve the Bayes estimates of $\Psi=\beta, \theta, R(t), H(t)$ or $C V(X)$ is given by

$$
\hat{\Psi}_{\mathrm{MC}}=\frac{1}{N-M} \sum_{i=M+1}^{N} \Psi^{(j)} .
$$

To calculate the CIs of $\Psi=\beta, \theta, R(t), H(t)$ and $C V(X)$, order $\beta^{(k)}, \theta^{(k)}, R^{(k)}(t), H^{(k)}(t)$ and $C V^{(k)}(X), k=1, \ldots, N$ as $\left\{\beta^{(1)}<\ldots<\beta^{(N)}\right\},\left\{\theta^{(1)}<\ldots<\theta^{(N)}\right\},\left\{R^{(1)}(t)<\ldots<R^{(N)}(t)\right\},\left\{H^{(1)}(t)<\ldots<H^{(N)}(t)\right\}$ and $\left\{C V^{(1)}(X)<\ldots<\right.$ $\left.C V^{(N)}(X)\right\}$. Thus, the $100(1-\tau) \%$ approximate CIs for $\beta, \theta, R(t), H(t)$ or $C V(X)$ become

$$
\left[\Psi_{\left(N \frac{\tau}{2}\right)}, \Psi_{\left(N\left(1-\frac{\tau}{2}\right)\right)}\right] .
$$

\section{Bayes estimation utilizing balanced loss functions}

Asymmetric loss function is often choosen, since it makes the statistical inferences more practical and applicable. Linearexponential loss function (see Varian [20]) and General entropy loss function (see Calabria et al. [21]) are the most well-known asymmetric loss functions. However, a more generalized loss function named the balanced loss function introduced by Jozani et al. [22] is given by

$$
L_{\rho, \omega, \delta_{0}}(\Theta, \delta)=\omega \rho\left(\delta, \delta_{0}\right)+(1-\omega) \rho(\Theta, \delta)
$$

where $\rho(\Theta, \delta)$ is an arbitrary loss function and $\delta_{0}$ is chosen as a prior 'target' estimator of $\Theta$, computed for the utilization of the standard of maximum likelihood, least-squares or unbiasedness. Depending on the observed value of $\delta_{0}(X), \operatorname{loss} L_{\rho, \omega, \delta_{0}}$ reflects a desire of closeness of $\delta$ to the target estimator $\delta_{0}$ and the unknown parameter $\Theta$. With the relative importance of these criteria governed by the choice of $0 \leq \omega<1 . L_{\rho, \omega, \delta_{0}}$ is specified to different choices of loss function for instance, for absolute value, balanced squared error loss (BSEL), balanced linear-exponential loss (BLINEXL) and balanced general entropy loss (BGEL) functions.

If we put $\rho(\Theta, \delta)=(\Theta-\delta)^{2}$ in Equation (5.1), it leads to BSEL function, see Ahmadi et al. [23], is

$$
L_{\omega, \delta_{0}}(\Theta, \delta)=\omega\left(\delta-\delta_{0}\right)^{2}+(1-\omega)(\delta-\Theta)^{2},
$$

then the Bayes estimator of the unknown parameter $\Theta$ based on BSEL is

$$
\delta_{\omega, \delta_{0}}(\underline{x})=\omega \delta_{0}(\underline{x})+(1-\omega) E(\Theta \mid \underline{x}) .
$$

Also, if we put $\rho(\Theta, \delta)=e^{c(\delta-h(\Theta))}-c(\Theta-\delta)-1$ with shape parameter $c, c \neq 0$ in Equation (5.1), it leads to BLINEXL function, see Zellner [24]. Thus, the Bayes estimation of $\Theta$ based on BLINEXL function is

$$
\delta_{\omega}(\underline{x})=\frac{-1}{c} \ln \left[\omega e^{-c \delta_{0}}+(1-\omega) E\left(e^{-c \Theta} \mid \underline{x}\right)\right] .
$$

In addition, the BGEL function with shape parameter $q$ is obtained with the choice of $\rho(\Theta, \delta)=\left(\frac{\delta}{\Theta}\right)^{q}-q \ln \left(\frac{\delta}{\Theta}\right)-1$. Thence, the Bayes estimation of $\Theta$ based on BGEL function is

$$
\delta_{\omega}(\underline{x})=\left[\omega\left(\delta_{0}\right)^{-q}+(1-\omega) E\left(\Theta^{-q} \mid \underline{x}\right)\right]^{\frac{-1}{q}} .
$$

The balanced loss functions are more comprehensive, since they include the MLE and both symmetric and asymmetric Bayes estimates as special cases. For instance, when $\omega=1$ in Equation (5.3) the Bayes estimate based on BSEL function limited 
to ML estimate and when $\omega=0$ it limited to the Bayes estimate relative to SEL function (symmetric). As well, the Bayes estimator based on BLINEXL function in (5.4) limited to ML estimate when $\omega=1$ and if $\omega=0$ it limited to the case of LINEXL function (asymmetric). Furthermore, the Bayes estimator based on BGEL function reduces to MLE if $\omega=1$ in

Equation (5.5) and when $\omega=0$, it reduces to the case of general entropy loss function. When $\Theta=(\beta, \theta, R(t), H(t), C V(X))$ and according to MCMC technique described above, the approximate posterior means under BSEL, BLINEXL and BGEL functions can be formulated, respectively, by

$$
\begin{gathered}
E[\Theta]=\omega \delta_{0}(\underline{x})+\frac{1-\omega}{N-M} \sum_{j=M+1}^{N} \Theta^{(j)}, \\
E[\Theta]=\frac{-1}{c} \ln \left[\omega e^{-c \delta_{0}(\underline{x})}+\frac{1-\omega}{N-M} \sum_{j=M+1}^{N} e^{-c \Theta^{(j)}}\right], \\
E[\Theta]=\left[\omega\left(\delta_{0}(\underline{x})\right)^{-q}+\frac{1-\omega}{N-M} \sum_{j=M+1}^{N}\left(\Theta^{(j)}\right)^{-q}\right]^{-q} .
\end{gathered}
$$

Subsequently, the approximate Bayes estimates for $\Theta=\beta, \theta, R(t), H(t)$ or $C V(X)$ based on BSEL, BLINEXL and BGEL functions can be computed, respectively, by

$$
\begin{gathered}
\hat{\Theta}_{\mathrm{BS}}=\omega \hat{\Theta}+\frac{1-\omega}{N-M} \sum_{j=M+1}^{N} \Theta^{(j)}, \\
\hat{\Theta}_{\mathrm{BL}}=\frac{-1}{c} \ln \left[\omega e^{-c \hat{\Theta}}+\frac{1-\omega}{N-M} \sum_{j=M+1}^{N} e^{-c \Theta^{(j)}}\right], \\
\hat{\Theta}_{\mathrm{BGE}}=\left[\omega(\hat{\Theta})^{-q}+\frac{1-\omega}{N-M} \sum_{j=M+1}^{N}\left(\Theta^{(j)}\right)^{-q}\right]^{-q} .
\end{gathered}
$$

\section{Application a set of real data}

We introduce an example utilizing a set of real data to explain the computations of the methods proposed in this article. The set of real data is extracted from Ghazal and Hasaballah [25]. The K-S test value is 0.0658573 of the EPD. From Figure 1 , it is clearly seen that the EPD is a good model fitting this data. To facilitate the computation processes, we have divided each data point by 10. According to unified HCS, we consider the six cases as following:

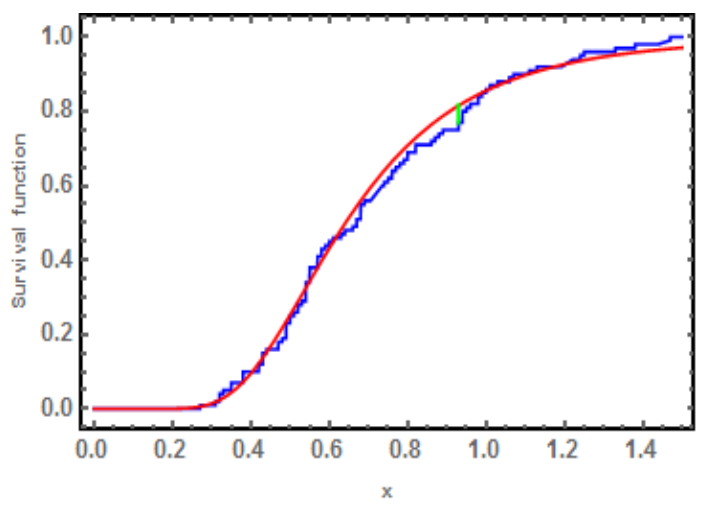

Figure 1: Empirical and Fitted Survival Functions. 
- Case I: $T_{1}=0.8, T_{2}=1.1, k=60, r=65 .\left(D=67, T^{*}=T_{1}=0.8\right)$.

- Case II: $T_{1}=0.8, T_{2}=1.1, k=60, r=70 .\left(D=70, T^{*}=x_{r: n}=0.82\right)$.

- Case III: $T_{1}=0.8, T_{2}=1.1, k=60, r=92 .\left(D=90, T^{*}=T_{2}=1.1\right)$.

- Case IV: $T_{1}=0.8, T_{2}=1.1, k=80, r=85$. $\left(D=85, T^{*}=x_{r: n}=0.99\right)$.

- Case V: $T_{1}=0.8, T_{2}=1.05, k=83, r=91$. $\left(D=88, T^{*}=T_{2}=1.05\right)$.

- Case VI: $T_{1}=0.6, T_{2}=1.05, k=93, r=94 .\left(D=93, T^{*}=x_{k: n}=1.2\right)$.

The MLEs for $\beta$ and $\theta$ of complete sample $(n=100)$ are $\hat{\beta}_{\mathrm{ML}}=7.45561$ and $\hat{\theta}_{\mathrm{ML}}=28.1245$. Also, using Equation (2.7) with $t=0.6$ the MLEs for $R(t)$ and $H(t)$ are $\hat{R}_{\mathrm{ML}}(t)=0.576303$ and $\hat{H}_{\mathrm{ML}}(t)=2.98727$, as well as the MLEs for $C V(X)$ is $\widehat{C V}_{\mathrm{ML}}(X)=0.459203$. Based on different methods (MLEs, Boot-p, Boot-t and MCMC) discussed in the above sections, we compute the point and interval estimations for $\beta, \theta, R(t), H(t)$ and $C V(X)$ under unified HCS. To calculate the Bayes estimates for $\beta, \theta, R(t), H(t)$ and $C V(X)$ against BSEL, BLINEXL and BGEL functions, we suppose the informative priors on both $\beta$ and $\theta$ as follows:

Case I $\left(a_{1}=133\right)$, Case II $\left(a_{1}=133\right)$, Case III $\left(a_{1}=225\right)$, Case IV $\left(a_{1}=205\right)$, Case V $\left(a_{1}=215\right)$ and Case VI $\left(a_{1}=240\right)$ and $b_{1}=a_{2}=b_{2}=0$ in the six cases. Using algorithm in Section 4 , we generate $N=16000 \mathrm{MCMC}$ samples $\left\{\left(\beta^{(j)}, \theta^{(j)}\right), j=\right.$ $1,2,3, \ldots, 16000\}$ with discarding the first $M=1000$ values as 'burn-in'. The MCMC findings of the posterior mean, median, mode, standard deviation (SD), standard errors (SE) and skewness for $\beta, \theta, R(t), H(t)$ and $C V(X)$ are reported in Table 1. The estimates and the corresponding $95 \%$ CIs of $\beta, \theta, R(t), H(t)$ and $C V(X)$ displayed in Tables 2 and 3. With $c=-7,0.3 .7$ and $q=-7,0.3 .7$, the findings of Bayes estimates relative to BSEL, BLINEXL and BGEL functions with various values of $w=0,0.2,0.5,0.9$ for $\beta, \theta, R(t), H(t)$ and $C V(X)$ are presented in Tables 4, 5, 6, 7, 8 and 9.

\section{Concluding remarks}

The aim of this article is to utilize various methods (MLEs, Boot-p, Boot-t and MCMC) to estimate and construct CIs of the parameters $\beta$ and $\theta$ also reliability, hazard functions and coefficient of variation of the exponentiated Pareto distribution based on unified hybrid censoring data. We assume that the gamma priors of $\beta$ and $\theta$ and the Bayes estimators based on the assumptions for balanced squared error loss, balanced linear-exponential loss and general entropy loss functions. In this article, the Bayes estimates cannot be computed in closed form. Thus, we utilize the MCMC method to calculate the approximate Bayes estimates and the corresponding CIs. Further, the details have been explained utilizing a real life data example.

\section{Acknowledgment}

The authors would like to thank the referees for their considerable suggestions and comments which contribute to improving the article eligibility.

\section{References}

[1] B. Epstein, Truncated life-test in exponential case, Ann. Math. Stat. 25 (1954) 555-564.

[2] A. Childs, B. Chandrasekar, N. Balakrishnan, D. Kundu, Exact likelihood inference based on Type-I and Type-II hybrid censored samples from the exponential distribution, Ann. Inst. Stat. Math. 55 (2003) 319-330.

[3] B. Chandrasekar, A. Childs, N. Balakrishnan, Exact likelihood inference for the exponential distribution under generalized Type-I and Type-II hybrid censoring, Nav. Res. Logist. 51 (2004) 994-1004.

[4] N. Balakrishnan, A. Rasouli, N. Sanjari Farsipour, Exact likelihood inference based on an unified hybrid censored sample from the exponential distribution, J. of Statist. Comput. and Simul. 78 (2008) 475-788. 
[5] N. Balakrishnan, D. Kundu, Hybrid censoring: models, inferential results and applications, Comput. Stat. Data Anal. 57 (2013) 166-209.

[6] H. Panahi, A. Sayyareh, Estimation and prediction for a unified hybrid-censored Burr Type XII distribution, J. Stat. Comput. Simul. 86 (2016) 55-73.

[7] A.R. Shafay, Exact inference for a simple step-stress model with generalized type-I hybrid censored data from the exponential distribution, Commun. Stat. Simu. Compu. 45 (2016) 181-206.

[8] M.A.W. Mahmoud, M.G.M. Ghazal, Estimations from the exponentiated rayleigh distribution based on generalized Type-II hybrid censored data, J. Egypt. Math. Soc. 25 (2017) 71-78.

[9] M.G.M. Ghazal, H.M. Hasaballah, Bayesian estimations using MCMC aapproach under exponentiated Rayleigh ristribution based on unified hybrid censored scheme, J. Stat. Appl. Pro. 6 (2017) 329-344.

[10] R.C. Gupta, R.D. Gupta, P.L. Gupta, Modeling failure time data by Lehman alternatives, Commun. Stat.-Theory Method. 27(4) (1998) 887-904.

[11] M.M. Ali, M. Pal, J. Woo, Some exponentiated distributions, Korean Commun. Stat. 14 (2007) 93-109.

[12] M.M. Ali, M. Pal, J. Woo, On the ratio of two independent exponentiated Pareto variables, Austrian J. Stat. 39(4) (2010) 329-340.

[13] A.I. Shawky, H.H. Abu-Zinadah, Exponentiated Pareto distribution: different method of estimations, Int. J. Contemp. Math. 14 (2009) 677-693.

[14] W.M. Afify, On estimation of the exponentiated Pareto distribution under different sample schemes, Stat. Method. 7 (2010) 77-83.

[15] M.A.W. Mahmoud, N.M. Yhiea, SH.M. El-Said, Estimation of parameters for the exponentiated Pareto distribution based on progressively type-II right censored data, J. Egypt. Math. Soc. 24 (2016) 431-436.

[16] K.K. Sharma, H. Krishna, Asymptotic sampling distribution of inverse coefficient of variation and its applications, IEEE Trans. Reliab. 43 (1994) 630-633.

[17] W.H. Greene, Econometric Analysis, Prentice Hall, New York, 2000.

[18] B.Efron,The bootstrap and other resampling plans. In: CBMS-NSF regional conference seriesin, applied mathematics. SIAM, Philadelphia, 1982.

[19] P. Hall, Theoretical comparison of Bootstrap confidence intervals, Ann Stat. 16 (1988) 927-953.

[20] H.R. Varian, A Bayesian Approach to Real Estate Assessment, North Holland, Amsterdam. (1975) 195-208.

[21] R. Calabria, G. Pulcini, Point estimation under asymmetric loss function for left truncated exponential samples, Commun. Stat.-Theory Mathod. 25 (1996) 585-600.

[22] M.J. Jozani, E. Marchand, A. Parsian, Bayes and robust Bayesian estimation under a general class of balanced loss functions, Stat. Pap. 53 (2012) 51-60.

[23] J. Ahmadi, M.J. Jozani, E. Marchand, A. Parsian, Bayes estimation based on krecord data from a general class of distributions under balanced type loss functions, J. Stat. Plan. Infer. 139 (2009) 1180-1189.

[24] A. Zellner, Bayesian estimation and prediction using asymmetric loss functions, J. Am. Stat. Assoc. 81 (1986) 446-451.

[25] M.G.M. Ghazal, H.M. Hasaballah, Exponentiated Rayleigh distribution: A Bayes study using MCMC approach based on unified hybrid censored data, J. Adv. Math. 12 (2017) 6863-6880. 
Table 1: MCMC findings for some posterior characteristics of six cases.

\begin{tabular}{|c|c|c|c|c|c|c|c|}
\hline & Parameters & Mean & Median & Mode & SD & $\mathrm{SE}$ & Skewness \\
\hline \multirow{5}{*}{ Case I } & $\beta$ & 6.861 & 6.8517 & 6.8331 & 0.4854 & 6.8782 & 0.1465 \\
\hline & $\theta$ & 22.7281 & 22.3002 & 21.4444 & 4.6592 & 23.2007 & 0.5576 \\
\hline & $R(t=0.6)$ & 0.5959 & 0.5951 & 0.5933 & 0.0938 & 0.6033 & -0.0086 \\
\hline & $H(t=0.6)$ & 2.6809 & 2.6864 & 2.6972 & 0.5019 & 2.7275 & -0.0352 \\
\hline & $C V(X)$ & 0.4964 & 0.4953 & 0.493 & 0.0263 & 0.4971 & 0.3936 \\
\hline \multirow{5}{*}{ Case II } & $\beta$ & 6.5621 & 6.5534 & 6.536 & 0.4581 & 6.5781 & 0.1035 \\
\hline & $\theta$ & 20.113 & 19.7736 & 19.0948 & 4.0566 & 20.518 & 0.4241 \\
\hline & $R(t=0.6)$ & 0.6036 & 0.6023 & 0.5995 & 0.0906 & 0.6104 & -0.0064 \\
\hline & $H(t=0.6)$ & 2.5437 & 2.5523 & 2.5695 & 0.4649 & 2.5858 & -0.0476 \\
\hline & $C V(X)$ & 0.5168 & 0.5152 & 0.5121 & 0.028 & 0.5176 & 0.5016 \\
\hline \multirow{5}{*}{ Case III } & $\beta$ & 7.115 & 7.1068 & 7.0906 & 0.3999 & 7.1262 & 0.1322 \\
\hline & $\theta$ & 24.9147 & 24.6865 & 24.23 & 4.4561 & 25.31 & 0.3945 \\
\hline & $R(t=0.6)$ & 0.5867 & 0.5859 & 0.5843 & 0.0799 & 0.5921 & 0.0154 \\
\hline & $H(t=0.6)$ & 2.8116 & 2.8177 & 2.8301 & 0.4264 & 2.8437 & -0.0515 \\
\hline & $C V(X)$ & 0.4803 & 0.4791 & 0.4768 & 0.0212 & 0.4808 & 0.4648 \\
\hline \multirow{5}{*}{ Case IV } & $\beta$ & 7.1156 & 7.1114 & 7.1032 & 0.4191 & 7.1279 & 0.0658 \\
\hline & $\theta$ & 24.9453 & 24.541 & 23.7324 & 4.4981 & 25.3476 & 0.4303 \\
\hline & $R(t=0.6)$ & 0.587 & 0.5859 & 0.5836 & 0.0822 & 0.5928 & 0.0053 \\
\hline & $H(t=0.6)$ & 2.8106 & 2.8192 & 2.8363 & 0.4435 & 2.8454 & -0.0463 \\
\hline & $C V(X)$ & 0.4803 & 0.479 & 0.4765 & 0.0215 & 0.4807 & 0.3962 \\
\hline \multirow{5}{*}{ Case V } & $\beta$ & 7.0745 & 7.067 & 7.0518 & 0.4071 & 7.0862 & 0.111 \\
\hline & $\theta$ & 24.7039 & 24.4841 & 24.0445 & 4.3868 & 25.0903 & 0.3756 \\
\hline & $R(t=0.6)$ & 0.5907 & 0.5895 & 0.5871 & 0.0804 & 0.5961 & 0.0228 \\
\hline & $H(t=0.6)$ & 2.7812 & 2.7883 & 2.8026 & 0.4314 & 2.8144 & -0.0534 \\
\hline & $C V(X)$ & 0.4821 & 0.4807 & 0.478 & 0.0213 & 0.4826 & 0.4176 \\
\hline \multirow{5}{*}{ Case VI } & $\beta$ & 7.1504 & 7.1427 & 7.1274 & 0.3923 & 7.1611 & 0.137 \\
\hline & $\theta$ & 25.1272 & 24.7544 & 24.0089 & 4.401 & 25.5097 & 0.4166 \\
\hline & $R(t=0.6)$ & 0.5839 & 0.583 & 0.5813 & 0.0788 & 0.5892 & 0.014 \\
\hline & $H(t=0.6)$ & 2.8358 & 2.8392 & 2.8459 & 0.4216 & 2.867 & -0.0485 \\
\hline & $C V(X)$ & 0.4785 & 0.4776 & 0.4758 & 0.0203 & 0.4789 & 0.3511 \\
\hline
\end{tabular}


Table 2: Estimates for $\beta, \theta, R(t), H(t)$ and $C V(X)$ under MLE, parametric bootstrap and MCMC technique of six cases.

\begin{tabular}{lcccccc}
\hline & Method & $\beta$ & $\theta$ & $R(t=0.6)$ & $H(t=0.6)$ & $C V(X)$ \\
\hline Case I & ML & 6.97144 & 23.1841 & 0.59029 & 2.03667 & 0.48754 \\
& Boot-p & 7.0952 & 24.8524 & 0.58239 & 2.82435 & 0.48319 \\
& Boot-t & 7.46417 & 24.5654 & 0.57049 & 3.07851 & 0.49106 \\
& MCMC & 6.85352 & 22.774 & 0.5985 & 2.66933 & 0.49623 \\
\hline Case II & ML & 7.05924 & 23.9767 & 0.5872 & 2.79556 & 0.48226 \\
& Boot-p & 7.10847 & 25.9136 & 0.58903 & 2.81192 & 0.48336 \\
& Boot-t & 7.00733 & 25.4001 & 0.59383 & 2.72784 & 0.46934 \\
& MCMC & 6.56213 & 20.113 & 0.60364 & 2.54371 & 0.51681 \\
\hline Case III & ML & 7.20674 & 25.448 & 0.58318 & 2.86623 & 0.47339 \\
& Boot-p & 7.25657 & 26.8257 & 0.58456 & 2.87815 & 0.47185 \\
& Boot-t & 7.36938 & 21.2201 & 0.58647 & 3.02731 & 0.53394 \\
& MCMC & 7.11497 & 24.9147 & 0.58669 & 2.81155 & 0.48028 \\
\hline Case IV & ML & 7.16195 & 25.0024 & 0.58455 & 2.84412 & 0.47601 \\
& Boot-p & 7.28059 & 27.2329 & 0.58389 & 2.89147 & 0.47107 \\
& Boot-t & 7.64466 & 26.9221 & 0.5905 & 3.19965 & 0.40925 \\
& MCMC & 7.11556 & 24.9453 & 0.58705 & 2.81059 & 0.48025 \\
\hline Case V & ML & 7.189 & 25.2702 & 0.58372 & 2.85748 & 0.47442 \\
& Boot-p & 7.26187 & 26.8987 & 0.58511 & 2.87826 & 0.47133 \\
& Boot-t & 7.30431 & 22.1696 & 0.58736 & 2.98266 & 0.51331 \\
& MCMC & 7.07455 & 24.7039 & 0.59067 & 2.78119 & 0.48208 \\
\hline Case VI & ML & 7.22595 & 25.6438 & 0.58263 & 2.8756 & 0.47226 \\
& Boot-p & 7.3335 & 27.7874 & 0.58172 & 2.92049 & 0.46819 \\
& Boot-t & 7.14026 & 23.969 & 0.5781 & 2.7977 & 0.52805 \\
& MCMC & 7.15036 & 25.1272 & 0.58392 & 2.83584 & 0.4785 \\
\hline
\end{tabular}


Table 3: $95 \%$ confidence lengths for $\beta, \theta, R(t), H(t)$ and $C V(X)$ under MLEs, parametric bootstrap and MCMC technique of six cases.

\begin{tabular}{|c|c|c|c|c|c|c|c|c|c|}
\hline \multirow[b]{2}{*}{ Cases } & \multirow[b]{2}{*}{ Parameters } & \multicolumn{2}{|c|}{ MLE } & \multicolumn{2}{|c|}{ Boot-p } & \multicolumn{2}{|c|}{ Boot-t } & \multicolumn{2}{|c|}{ MCMC } \\
\hline & & Lower & Upper & Lower & Upper & Lower & Upper & Lower & Upper \\
\hline \multirow{5}{*}{ Case I } & $\beta$ & 5.57444 & 8.36845 & 6.28984 & 8.13133 & 7.08997 & 7.88393 & 5.94551 & 7.84689 \\
\hline & $\theta$ & 9.25782 & 37.1103 & 15.6495 & 41.3592 & 21.8757 & 26.8198 & 14.6674 & 32.9259 \\
\hline & $R(t=0.6)$ & 0.50942 & 0.67115 & 0.53016 & 0.63142 & 0.49261 & 0.63629 & 0.3995 & 0.78791 \\
\hline & $H(t=0.6)$ & 2.03667 & 3.46563 & 2.52776 & 3.17531 & 2.96291 & 3.26006 & 1.65458 & 3.70527 \\
\hline & $C V(X)$ & 0.3979 & 0.57719 & 0.41706 & 0.54645 & 0.32684 & 0.70127 & 0.44953 & 0.55235 \\
\hline \multirow{5}{*}{ Case II } & $\beta$ & 5.68382 & 8.43466 & 5.67865 & 8.7106 & 6.52859 & 7.45695 & 5.69059 & 7.47855 \\
\hline & $\theta$ & 9.77644 & 38.1771 & 13.8901 & 48.61 & 22.2252 & 27.8813 & 12.7653 & 29.1825 \\
\hline & $R(t=0.6)$ & 0.50678 & 0.66763 & 0.49853 & 0.67581 & 0.50402 & 0.6903 & 0.41843 & 0.78841 \\
\hline & $H(t=0.6)$ & 2.08686 & 3.50425 & 2.10467 & 3.62305 & 2.53351 & 2.91348 & 1.58963 & 3.47845 \\
\hline & $C V(X)$ & 0.39594 & 0.56857 & 0.39821 & 0.58255 & 0.23724 & 0.72332 & 0.46647 & 0.57734 \\
\hline \multirow{5}{*}{ Case III } & $\beta$ & 5.97634 & 8.43714 & 6.37307 & 8.2871 & 7.10278 & 7.65482 & 6.3573 & 7.91398 \\
\hline & $\theta$ & 11.7262 & 39.1698 & 17.2515 & 40.9707 & 19.0619 & 23.1343 & 16.7529 & 34.4034 \\
\hline & $R(t=0.6)$ & 0.5051 & 0.66126 & 0.51404 & 0.65463 & 0.51373 & 0.66256 & 0.42672 & 0.7514 \\
\hline & $H(t=0.6)$ & 2.21839 & 3.51407 & 2.42478 & 3.39854 & 2.89376 & 3.15228 & 1.93355 & 3.65404 \\
\hline & $C V(X)$ & 0.39852 & 0.54826 & 0.41568 & 0.52986 & 0.3998 & 0.68004 & 0.44239 & 0.52669 \\
\hline \multirow{5}{*}{ Case IV } & $\beta$ & 5.90362 & 8.42027 & 6.25235 & 8.46247 & 7.30592 & 7.99233 & 6.3058 & 7.93951 \\
\hline & $\theta$ & 11.2562 & 38.7486 & 16.6775 & 44.2927 & 24.1808 & 29.4337 & 16.636 & 34.5881 \\
\hline & $R(t=0.6)$ & 0.50609 & 0.66301 & 0.50384 & 0.65875 & 0.51659 & 0.67767 & 0.41951 & 0.75514 \\
\hline & $H(t=0.6)$ & 2.18582 & 3.50241 & 2.36907 & 3.55776 & 3.02233 & 3.35233 & 1.90981 & 3.70613 \\
\hline & $C V(X)$ & 0.39865 & 0.55337 & 0.40656 & 0.53931 & 0.25995 & 0.57195 & 0.44056 & 0.52678 \\
\hline \multirow{5}{*}{ Case V } & $\beta$ & 5.94792 & 8.43008 & 6.41227 & 8.22615 & 7.04456 & 7.5644 & 6.30024 & 7.89359 \\
\hline & $\theta$ & 11.5412 & 38.9991 & 17.5747 & 41.9445 & 20.0557 & 24.0727 & 16.6075 & 34.1971 \\
\hline & $R(t=0.6)$ & 0.5055 & 0.66194 & 0.51543 & 0.65841 & 0.51421 & 0.66318 & 0.42847 & 0.75636 \\
\hline & $H(t=0.6)$ & 2.20572 & 3.50925 & 2.40971 & 3.42833 & 2.85774 & 3.094 & 1.89419 & 3.64812 \\
\hline & $C V(X)$ & 0.39859 & 0.55025 & 0.41601 & 0.52875 & 0.3904 & 0.65372 & 0.44349 & 0.5276 \\
\hline \multirow{5}{*}{ Case VI } & $\beta$ & 6.01103 & 8.44088 & 6.32234 & 8.56298 & 6.8357 & 7.471 & 6.40075 & 7.93886 \\
\hline & $\theta$ & 11.9676 & 39.32 & 17.1728 & 46.1368 & 21.3695 & 26.308 & 17.1935 & 34.6209 \\
\hline & $R(t=0.6)$ & 0.50473 & 0.66052 & 0.50331 & 0.65522 & 0.50349 & 0.657 & 0.42343 & 0.74704 \\
\hline & $H(t=0.6)$ & 2.23369 & 3.51751 & 2.38698 & 3.58632 & 2.65494 & 2.94333 & 1.96199 & 3.68566 \\
\hline & $C V(X)$ & 0.39865 & 0.54588 & 0.40205 & 0.53428 & 0.36355 & 0.71378 & 0.44135 & 0.52132 \\
\hline
\end{tabular}


Table 4: Bayes MCMC estimates based on BSEL, BLINEXL and BGEL functions for Case I.

\begin{tabular}{|c|c|c|c|c|c|c|c|c|}
\hline \multirow[b]{2}{*}{ Parameters } & \multirow[b]{2}{*}{$\omega$} & \multirow[t]{2}{*}{ BSEL } & \multicolumn{3}{|c|}{ BLINEXL } & \multicolumn{3}{|c|}{ BGEL } \\
\hline & & & $\mathrm{c}=-7$ & $\mathrm{c}=0.3$ & $c=7$ & $q=-7$ & $q=0.3$ & $q=7$ \\
\hline \multirow[t]{4}{*}{$\beta$} & 0.0 & 6.86103 & 7.90801 & 6.82593 & 6.16709 & 6.96378 & 6.83872 & 6.72357 \\
\hline & 0.2 & 6.88311 & 7.87618 & 6.85453 & 6.19884 & 6.96532 & 6.865 & 6.76771 \\
\hline & 0.5 & 6.91623 & 7.80919 & 6.89789 & 6.2656 & 6.96762 & 6.90466 & 6.83856 \\
\hline & 0.9 & 6.9604 & 7.58088 & 6.9566 & 6.49149 & 6.97068 & 6.95802 & 6.94318 \\
\hline \multirow[t]{4}{*}{$\theta$} & 0.0 & 22.7281 & 53.3899 & 20.0002 & 10.9066 & 25.7905 & 22.1154 & 18.6907 \\
\hline & 0.2 & 22.8193 & 53.3581 & 20.4379 & 10.9385 & 25.3845 & 22.3239 & 19.1482 \\
\hline & 0.5 & 22.9561 & 53.2909 & 21.2256 & 11.0056 & 24.6911 & 22.6415 & 20.0551 \\
\hline & 0.9 & 23.1385 & 53.061 & 22.6896 & 11.2355 & 23.5347 & 23.0742 & 22.2069 \\
\hline \multirow[t]{4}{*}{$R(t=0.6)$} & 0.0 & 0.59595 & 0.62711 & 0.59463 & 0.56464 & 0.63702 & 0.58587 & 0.51361 \\
\hline & 0.2 & 0.59482 & 0.62047 & 0.59376 & 0.56942 & 0.62921 & 0.58675 & 0.52346 \\
\hline & 0.5 & 0.59312 & 0.60988 & 0.59246 & 0.57689 & 0.61629 & 0.58807 & 0.54171 \\
\hline & 0.9 & 0.59085 & 0.59443 & 0.59072 & 0.5875 & 0.59606 & 0.58984 & 0.57756 \\
\hline \multirow[t]{4}{*}{$H(t=0.6)$} & 0.0 & 2.68093 & 3.63633 & 2.64304 & 1.63225 & 2.93443 & 2.61466 & 1.49219 \\
\hline & 0.2 & 2.69498 & 3.60453 & 2.66439 & 1.66412 & 2.90298 & 2.64124 & 1.53976 \\
\hline & 0.5 & 2.71604 & 3.5376 & 2.69666 & 1.73122 & 2.85159 & 2.68178 & 1.64429 \\
\hline & 0.9 & 2.74413 & 3.30999 & 2.74018 & 1.96068 & 2.77304 & 2.73709 & 2.03898 \\
\hline \multirow[t]{4}{*}{$C V(X)$} & 0.0 & 0.49641 & 0.49889 & 0.49631 & 0.49405 & 0.50069 & 0.49552 & 0.49105 \\
\hline & 0.2 & 0.49464 & 0.49669 & 0.49455 & 0.49273 & 0.49822 & 0.49391 & 0.49033 \\
\hline & 0.5 & 0.49198 & 0.49333 & 0.49192 & 0.49076 & 0.49438 & 0.49151 & 0.48927 \\
\hline & 0.9 & 0.48843 & 0.48872 & 0.48842 & 0.48818 & 0.48896 & 0.48833 & 0.48789 \\
\hline
\end{tabular}


Table 5: Bayes MCMC estimates based on BSEL, BLINEXL and BGEL functions for Case II.

\begin{tabular}{|c|c|c|c|c|c|c|c|c|}
\hline \multirow[b]{2}{*}{ Parameters } & \multirow[b]{2}{*}{$\omega$} & \multirow[t]{2}{*}{ BSEL } & \multicolumn{3}{|c|}{ BLINEXL } & \multicolumn{3}{|c|}{ BGEL } \\
\hline & & & $c=-7$ & $\mathrm{c}=0.3$ & $c=7$ & $q=-7$ & $q=0.3$ & $q=7$ \\
\hline \multirow[t]{4}{*}{$\bar{\beta}$} & 0.0 & 6.56213 & 7.32888 & 6.5308 & 5.94403 & 6.65731 & 6.54131 & 6.43332 \\
\hline & 0.2 & 6.66155 & 7.30231 & 6.63 & 5.97589 & 6.74986 & 6.64084 & 6.52632 \\
\hline & 0.5 & 6.81069 & 7.25001 & 6.78456 & 6.04299 & 6.87583 & 6.79387 & 6.68924 \\
\hline & 0.9 & 7.00953 & 7.12279 & 7.00247 & 6.27245 & 7.0248 & 7.0051 & 6.97146 \\
\hline \multirow[t]{4}{*}{$\theta$} & 0.0 & 20.113 & 35.4447 & 17.9547 & 8.44189 & 22.5324 & 19.581 & 16.4863 \\
\hline & 0.2 & 20.8857 & 35.4128 & 18.5644 & 8.47377 & 22.8678 & 20.3706 & 16.9765 \\
\hline & 0.5 & 22.0449 & 35.3457 & 19.7583 & 8.54091 & 23.3214 & 21.6344 & 18.0208 \\
\hline & 0.9 & 23.5904 & 35.1158 & 22.6053 & 8.77083 & 23.8541 & 23.4828 & 21.3187 \\
\hline \multirow[t]{4}{*}{$R(t=0.6)$} & 0.0 & 0.60364 & 0.63254 & 0.60241 & 0.57462 & 0.64152 & 0.59443 & 0.53311 \\
\hline & 0.2 & 0.60035 & 0.62455 & 0.59936 & 0.57705 & 0.6327 & 0.59298 & 0.54105 \\
\hline & 0.5 & 0.59542 & 0.61166 & 0.5948 & 0.58077 & 0.61791 & 0.5908 & 0.55503 \\
\hline & 0.9 & 0.58885 & 0.59244 & 0.58872 & 0.58589 & 0.59415 & 0.58792 & 0.57951 \\
\hline \multirow[t]{4}{*}{$H(t=0.6)$} & 0.0 & 2.54371 & 3.29342 & 2.5112 & 1.72334 & 2.77178 & 2.48433 & 1.83342 \\
\hline & 0.2 & 2.59408 & 3.26263 & 2.56616 & 1.7552 & 2.77664 & 2.54283 & 1.88931 \\
\hline & 0.5 & 2.66963 & 3.19871 & 2.65035 & 1.82229 & 2.78382 & 2.63398 & 2.0096 \\
\hline & 0.9 & 2.77037 & 2.99928 & 2.766 & 2.05158 & 2.79323 & 2.76223 & 2.41119 \\
\hline \multirow[t]{4}{*}{$C V(X)$} & 0.0 & 0.51681 & 0.51965 & 0.51669 & 0.51415 & 0.52152 & 0.51584 & 0.51105 \\
\hline & 0.2 & 0.5099 & 0.51291 & 0.50978 & 0.50718 & 0.51499 & 0.50888 & 0.50413 \\
\hline & 0.5 & 0.49953 & 0.50217 & 0.49943 & 0.49732 & 0.50417 & 0.49868 & 0.49499 \\
\hline & 0.9 & 0.48571 & 0.48647 & 0.48568 & 0.48514 & 0.48713 & 0.48549 & 0.4846 \\
\hline
\end{tabular}


Table 6: Bayes MCMC estimates based on BSEL, BLINEXL and BGEL functions for Case III.

\begin{tabular}{|c|c|c|c|c|c|c|c|c|}
\hline \multirow[b]{2}{*}{ Parameters } & \multirow[b]{2}{*}{$\omega$} & \multirow[t]{2}{*}{ BSEL } & \multicolumn{3}{|c|}{ BLINEXL } & \multicolumn{3}{|c|}{ BGEL } \\
\hline & & & $c=-7$ & $\mathrm{c}=0.3$ & $c=7$ & $q=-7$ & $q=0.3$ & $q=7$ \\
\hline \multirow[t]{4}{*}{$\beta$} & 0.0 & 7.11497 & 7.73853 & 7.09111 & 6.61629 & 7.1824 & 7.10037 & 7.02519 \\
\hline & 0.2 & 7.13333 & 7.70751 & 7.11391 & 6.64759 & 7.18731 & 7.12148 & 7.05864 \\
\hline & 0.5 & 7.16086 & 7.64292 & 7.14842 & 6.71304 & 7.19463 & 7.1533 & 7.11134 \\
\hline & 0.9 & 7.19756 & 7.43771 & 7.19499 & 6.92597 & 7.20433 & 7.19601 & 7.18683 \\
\hline \multirow[t]{4}{*}{$\theta$} & 0.0 & 24.9147 & 45.9819 & 22.2421 & 11.6296 & 27.3333 & 24.395 & 21.2545 \\
\hline & 0.2 & 25.0213 & 45.95 & 22.6817 & 11.6615 & 27.015 & 24.601 & 21.7293 \\
\hline & 0.5 & 25.1813 & 45.8829 & 23.4736 & 11.7286 & 26.4907 & 24.9142 & 22.6448 \\
\hline & 0.9 & 25.3947 & 45.653 & 24.9486 & 11.9586 & 25.6777 & 25.3401 & 24.6419 \\
\hline \multirow[t]{4}{*}{$R(t=0.6)$} & 0.0 & 0.58669 & 0.60928 & 0.58573 & 0.56416 & 0.61758 & 0.57937 & 0.53056 \\
\hline & 0.2 & 0.58598 & 0.60443 & 0.58522 & 0.56777 & 0.61158 & 0.58013 & 0.53834 \\
\hline & 0.5 & 0.58493 & 0.59683 & 0.58445 & 0.57336 & 0.60185 & 0.58127 & 0.55199 \\
\hline & 0.9 & 0.58353 & 0.58602 & 0.58344 & 0.58116 & 0.58721 & 0.5828 & 0.57576 \\
\hline \multirow[t]{4}{*}{$H(t=0.6)$} & 0.0 & 2.81155 & 3.50086 & 2.78421 & 2.119 & 2.9907 & 2.76737 & 2.45064 \\
\hline & 0.2 & 2.82249 & 3.4694 & 2.80045 & 2.15068 & 2.9682 & 2.78678 & 2.5012 \\
\hline & 0.5 & 2.83889 & 3.40351 & 2.82497 & 2.21725 & 2.93242 & 2.81623 & 2.59659 \\
\hline & 0.9 & 2.86076 & 3.1863 & 2.85793 & 2.44122 & 2.88021 & 2.85614 & 2.79275 \\
\hline \multirow[t]{4}{*}{$C V(X)$} & 0.0 & 0.48028 & 0.48189 & 0.48022 & 0.47875 & 0.48317 & 0.47968 & 0.4767 \\
\hline & 0.2 & 0.4789 & 0.48023 & 0.47885 & 0.47766 & 0.48131 & 0.47842 & 0.47602 \\
\hline & 0.5 & 0.47684 & 0.4777 & 0.4768 & 0.47604 & 0.47843 & 0.47652 & 0.47502 \\
\hline & 0.9 & 0.47408 & 0.47426 & 0.47407 & 0.47391 & 0.47442 & 0.47401 & 0.47371 \\
\hline
\end{tabular}


Table 7: Bayes MCMC estimates based on BSEL, BLINEXL and BGEL functions for Case IV.

\begin{tabular}{|c|c|c|c|c|c|c|c|c|}
\hline \multirow[b]{2}{*}{ Parameters } & \multirow[b]{2}{*}{$\omega$} & \multirow[t]{2}{*}{ BSEL } & \multicolumn{3}{|c|}{ BLINEXL } & \multicolumn{3}{|c|}{ BGEL } \\
\hline & & & $\mathrm{c}=-7$ & $\mathrm{c}=0.3$ & $\mathrm{c}=7$ & $q=-7$ & $q=0.3$ & $q=7$ \\
\hline \multirow[t]{4}{*}{$\beta$} & 0.0 & 7.11556 & 7.72509 & 7.08929 & 6.56409 & 7.18904 & 7.09948 & 7.01587 \\
\hline & 0.2 & 7.12484 & 7.6939 & 7.1037 & 6.59542 & 7.18367 & 7.11192 & 7.04322 \\
\hline & 0.5 & 7.13875 & 7.62881 & 7.12542 & 6.66095 & 7.17557 & 7.13063 & 7.0859 \\
\hline & 0.9 & 7.15731 & 7.41915 & 7.15461 & 6.87469 & 7.16469 & 7.15567 & 7.14621 \\
\hline \multirow[t]{4}{*}{$\theta$} & 0.0 & 24.9453 & 44.2832 & 22.3306 & 11.377 & 27.3889 & 24.4219 & 21.557 \\
\hline & 0.2 & 24.9567 & 44.2513 & 22.72 & 11.4088 & 27.0039 & 24.5366 & 21.9871 \\
\hline & 0.5 & 24.9738 & 44.1842 & 23.4056 & 11.476 & 26.3563 & 24.7099 & 22.7919 \\
\hline & 0.9 & 24.9966 & 43.9542 & 24.616 & 11.7059 & 25.3097 & 24.9435 & 24.4112 \\
\hline \multirow[t]{4}{*}{$R(t=0.6)$} & 0.0 & 0.58705 & 0.61092 & 0.58604 & 0.56318 & 0.61955 & 0.5793 & 0.52762 \\
\hline & 0.2 & 0.58655 & 0.60602 & 0.58574 & 0.56721 & 0.61345 & 0.58034 & 0.53583 \\
\hline & 0.5 & 0.5858 & 0.59835 & 0.58529 & 0.57347 & 0.60357 & 0.58192 & 0.55038 \\
\hline & 0.9 & 0.5848 & 0.58742 & 0.5847 & 0.58226 & 0.58866 & 0.58402 & 0.57628 \\
\hline \multirow[t]{4}{*}{$H(t=0.6)$} & 0.0 & 2.81059 & 3.55008 & 2.781 & 2.03854 & 3.00386 & 2.76255 & 2.38411 \\
\hline & 0.2 & 2.81729 & 3.51845 & 2.79353 & 2.07029 & 2.97581 & 2.77862 & 2.43678 \\
\hline & 0.5 & 2.82735 & 3.45207 & 2.81241 & 2.13705 & 2.9305 & 2.80295 & 2.538 \\
\hline & 0.9 & 2.84076 & 3.23003 & 2.83775 & 2.36298 & 2.86268 & 2.83582 & 2.75683 \\
\hline \multirow[t]{4}{*}{$C V(X)$} & 0.0 & 0.48025 & 0.4819 & 0.48018 & 0.47867 & 0.4832 & 0.47963 & 0.47653 \\
\hline & 0.2 & 0.4794 & 0.48074 & 0.47935 & 0.47813 & 0.48181 & 0.47891 & 0.47643 \\
\hline & 0.5 & 0.47813 & 0.47899 & 0.47809 & 0.47733 & 0.47969 & 0.47782 & 0.47627 \\
\hline & 0.9 & 0.47643 & 0.47661 & 0.47643 & 0.47627 & 0.47676 & 0.47637 & 0.47606 \\
\hline
\end{tabular}


Table 8: Bayes MCMC estimates based on BSEL, BLINEXL and BGEL functions for Case V.

\begin{tabular}{|c|c|c|c|c|c|c|c|c|}
\hline \multirow[b]{2}{*}{ Parameters } & \multirow[b]{2}{*}{$\omega$} & \multirow[t]{2}{*}{ BSEL } & \multicolumn{3}{|c|}{ BLINEXL } & \multicolumn{3}{|c|}{ BGEL } \\
\hline & & & $c=-7$ & $\mathrm{c}=0.3$ & $c=7$ & $q=-7$ & $q=0.3$ & $q=7$ \\
\hline \multirow[t]{4}{*}{$\beta$} & 0.0 & 7.07455 & 7.75339 & 7.04981 & 6.54455 & 7.14464 & 7.05932 & 6.98056 \\
\hline & 0.2 & 7.09744 & 7.7222 & 7.07718 & 6.57603 & 7.15364 & 7.08501 & 7.0185 \\
\hline & 0.5 & 7.13177 & 7.6571 & 7.11868 & 6.64201 & 7.16702 & 7.12378 & 7.07866 \\
\hline & 0.9 & 7.17755 & 7.44727 & 7.17481 & 6.86002 & 7.18463 & 7.17589 & 7.16581 \\
\hline \multirow[t]{4}{*}{$\theta$} & 0.0 & 24.7039 & 41.9896 & 22.1605 & 12.318 & 27.0212 & 24.1977 & 21.361 \\
\hline & 0.2 & 24.8171 & 41.9578 & 22.5916 & 12.3499 & 26.7224 & 24.4074 & 21.8201 \\
\hline & 0.5 & 24.987 & 41.8906 & 23.3652 & 12.417 & 26.2329 & 24.7264 & 22.696 \\
\hline & 0.9 & 25.2135 & 41.6607 & 24.7922 & 12.647 & 25.4808 & 25.1602 & 24.5501 \\
\hline \multirow[t]{4}{*}{$R(t=0.6)$} & 0.0 & 0.59067 & 0.61361 & 0.5897 & 0.56786 & 0.62182 & 0.58331 & 0.53368 \\
\hline & 0.2 & 0.58928 & 0.60811 & 0.5885 & 0.5709 & 0.61526 & 0.5834 & 0.54119 \\
\hline & 0.5 & 0.58719 & 0.59944 & 0.58671 & 0.57557 & 0.60456 & 0.58352 & 0.55429 \\
\hline & 0.9 & 0.58441 & 0.587 & 0.58432 & 0.58205 & 0.58825 & 0.58368 & 0.57678 \\
\hline \multirow[t]{4}{*}{$H(t=0.6)$} & 0.0 & 2.78119 & 3.5998 & 2.75319 & 2.06942 & 2.9665 & 2.73528 & 2.39518 \\
\hline & 0.2 & 2.79645 & 3.56812 & 2.77379 & 2.10115 & 2.94656 & 2.75917 & 2.4481 \\
\hline & 0.5 & 2.81934 & 3.50157 & 2.80493 & 2.16787 & 2.91505 & 2.79551 & 2.54982 \\
\hline & 0.9 & 2.84985 & 3.27781 & 2.84691 & 2.39328 & 2.86956 & 2.84495 & 2.76974 \\
\hline \multirow[t]{4}{*}{$C V(X)$} & 0.0 & 0.48208 & 0.48371 & 0.48201 & 0.48052 & 0.48498 & 0.48147 & 0.47844 \\
\hline & 0.2 & 0.48055 & 0.4819 & 0.48049 & 0.47928 & 0.48297 & 0.48005 & 0.47762 \\
\hline & 0.5 & 0.47825 & 0.47914 & 0.47822 & 0.47744 & 0.47987 & 0.47793 & 0.4764 \\
\hline & 0.9 & 0.47519 & 0.47538 & 0.47518 & 0.47502 & 0.47554 & 0.47512 & 0.47481 \\
\hline
\end{tabular}


Table 9: Bayes MCMC estimates based on BSEL, BLINEXL and BGEL functions for Case VI.

\begin{tabular}{|c|c|c|c|c|c|c|c|c|}
\hline \multirow[b]{2}{*}{ Parameters } & \multirow[b]{2}{*}{$\omega$} & \multirow[t]{2}{*}{ BSEL } & \multicolumn{3}{|c|}{ BLINEXL } & \multicolumn{3}{|c|}{ BGEL } \\
\hline & & & $\mathrm{c}=-7$ & $\mathrm{c}=0.3$ & $\mathrm{c}=7$ & $q=-7$ & $q=0.3$ & $q=7$ \\
\hline \multirow[t]{4}{*}{$\beta$} & 0.0 & 7.15036 & 7.73126 & 7.12739 & 6.66774 & 7.21497 & 7.13638 & 7.06448 \\
\hline & 0.2 & 7.16548 & 7.70042 & 7.14687 & 6.6989 & 7.21718 & 7.15418 & 7.09451 \\
\hline & 0.5 & 7.18816 & 7.63634 & 7.17631 & 6.76392 & 7.22048 & 7.18099 & 7.14157 \\
\hline & 0.9 & 7.21839 & 7.43555 & 7.21597 & 6.97294 & 7.22486 & 7.21693 & 7.20843 \\
\hline \multirow[t]{4}{*}{$\theta$} & 0.0 & 25.1272 & 43.1342 & 22.5886 & 11.0114 & 27.4518 & 24.629 & 21.8384 \\
\hline & 0.2 & 25.2305 & 43.1023 & 23.0148 & 11.0433 & 27.1442 & 24.8277 & 22.2957 \\
\hline & 0.5 & 25.3855 & 43.0352 & 23.7778 & 11.1105 & 26.6394 & 25.1297 & 23.1619 \\
\hline & 0.9 & 25.5921 & 42.8053 & 25.1777 & 11.3404 & 25.862 & 25.5399 & 24.9613 \\
\hline \multirow[t]{4}{*}{$R(t=0.6)$} & 0.0 & 0.58392 & 0.60594 & 0.58299 & 0.56198 & 0.6142 & 0.57677 & 0.52961 \\
\hline & 0.2 & 0.58366 & 0.60158 & 0.58292 & 0.56588 & 0.60863 & 0.57794 & 0.53742 \\
\hline & 0.5 & 0.58327 & 0.59476 & 0.58281 & 0.57193 & 0.59966 & 0.57969 & 0.55116 \\
\hline & 0.9 & 0.58276 & 0.58514 & 0.58266 & 0.58042 & 0.58628 & 0.58204 & 0.57512 \\
\hline \multirow[t]{4}{*}{$H(t=0.6)$} & 0.0 & 2.83584 & 3.50885 & 2.80911 & 2.09672 & 3.01039 & 2.79303 & 2.45969 \\
\hline & 0.2 & 2.84379 & 3.47739 & 2.82231 & 2.12845 & 2.98622 & 2.8093 & 2.51035 \\
\hline & 0.5 & 2.85572 & 3.41151 & 2.84219 & 2.19513 & 2.94761 & 2.83393 & 2.6059 \\
\hline & 0.9 & 2.87163 & 3.19442 & 2.86889 & 2.42025 & 2.89089 & 2.8672 & 2.80217 \\
\hline \multirow[t]{4}{*}{$C V(X)$} & 0.0 & 0.4785 & 0.47997 & 0.47844 & 0.47708 & 0.48113 & 0.47794 & 0.47514 \\
\hline & 0.2 & 0.47725 & 0.47846 & 0.4772 & 0.4761 & 0.47944 & 0.4768 & 0.47455 \\
\hline & 0.5 & 0.47538 & 0.47617 & 0.47535 & 0.47465 & 0.47682 & 0.47509 & 0.47368 \\
\hline & 0.9 & 0.47289 & 0.47305 & 0.47288 & 0.47274 & 0.4732 & 0.47283 & 0.47255 \\
\hline
\end{tabular}

